\title{
Dietary Habits and Nutrient Intakes of a Cohort of Healthy Children in Spain
}

\author{
Liliana Oliveros Leal ${ }^{1}$, Rocío Ramos Paúl Salto², Luis Torres Cardona ${ }^{2}$, Jennifer A. Williams*3 , \\ Barbara J. Marriage $^{3}$, Roberto Ruiz Debeza ${ }^{4}$, Enrique Conde Bueso ${ }^{5}$, Jorge del Campo Yubero ${ }^{5}$ \\ and Luis Ros Mar
}

${ }^{1}$ Hospital Universitario 12 de Octubre, Madrid, Spain
${ }^{2}$ BIEM Center of Psychology, Madrid, Spain
${ }^{3}$ Abbott Nutrition, Columbus, Ohio, USA
${ }^{4}$ Abbott Nutrition, Madrid, Spain
${ }^{5}$ Effice Research, Madrid, Spain
${ }^{6}$ Hospital Universitario Miguel Servet, Zaragoza, Spain

Abstract: Picky eating behavior is a common complaint among parents. Children labeled as "picky eaters" usually demonstrate behaviors of highly selective intake, by avoiding certain foods or eating a limited menu. The possibility for shortor long-term consequences as a result of picky eating behavior exists.

A cross-sectional survey study was performed on 1101 children from 3 to 6 years in seven schools of Madrid, Spain. Picky eaters (PE) were defined as children whose average consumption was $<65 \%$ of the recommended daily intake for 4 of 6 food groups. This study was designed to analyze the nutrient intakes of children and determine possible effects of eating behaviors on attention. Using the study definition, $18.4 \%$ of the sample was classified as PE. No differences were observed between the two groups for the attention measurement when analyzed as a whole. However, as the age of the children increased from 49 months on, the percentage of PE children with a negative score increased until 50\% in the oldest group. The PE group had lower energy and macronutrient levels, but managed to meet dietary recommendations for most micronutrients. Additionally, the PE children could be characterized by specific eating behaviors compared with the healthy eater group of children.

Keywords: Picky eating, eating behavior, attention, nutrient intake.

\section{INTRODUCTION}

Children labeled as "picky eaters" usually demonstrate behaviors of highly selective intake, by avoiding certain foods or only eating a limited number of foods [1]. Picky eaters are typically ages 3 years and older and do not exhibit severe growth problems. Estimates for picky eating in the general population vary widely, from estimates of $20-60 \%$ of all children. Feeding problems increase with age and the actual prevalence is highly dependent on the types of questions asked and parental perceptions and/or concerns. For example, a survey conducted in Europe using the question "Would you say your $x$ year old child is a 'picky eater'?" resulted in more than half of the 4-5 year olds being qualified as a picky eater. One reason for variation in the prevalence of picky eating behaviors among studies is the differences in age ranges of the children who are studied [1].

"Address correspondence to this author at Abbott Nutrition, 3300 Stelzer, Road Columbus, Ohio, 43219 USA; Tel: 614-624-3933;

Fax: 614-727-3933; E-mail: Jennifer.williams@abbott.com
The possibility for short- or long-term consequences as a result of picky eating behavior does exist. Short-term effects of picky eating can be nutritional deficiencies, such as low levels of energy and protein or micronutrients. Some picky eating children have demonstrated a sub-optimal consumption of nutrients such as lower protein, energy and fat intake; consumption of fewer than recommended fruits, vegetables and meats; or not meeting daily recommendations for specific vitamins and minerals [2-4]. Additionally, another short-term consequence for picky eating behavior may be growth limitations as some research has shown picky eating children to be shorter in stature and lighter in body weight $[5,6]$.

One possible long-term consequence of picky eating behavior is decreased cognitive performance. Scientific evidence links malnutrition with a deficit in cognitive development, and Kar et al, report that "children with poor diets have difficulties in tests involving attention, memory, visual perception, verbal understanding and other major cognitive processes" [7]. Even in children of developed countries, there 
is a relationship between picky eating and poor school performance [8-10]. Researchers have found the Mental Development Index scores of picky eaters to be significantly lower than those for healthy eating children in a cohort of toddlers [9].

The objective of the present multi-center cross-sectional survey study was to determine if there were any differences between PE children and children with healthy eating behaviors (HE) on measures of attention in a school setting and to compare their energy and nutrient intakes based on a threeday food record.

\section{MATERIALS AND METHODOLOGY}

\section{Participants}

2428 children between 36 and 78 months of age were recruited from seven private schools in Madrid, Spain. By assuming a prevalence of picky eating of $25 \%$ in the study population and a $10 \%$ difference in Cumanin scores between the two groups of subjects, a sample size of approximately 918 children, 734 with healthy eating habits and 184 with picky eating behaviors, was estimated to have a 95\% confidence level and a power of $80 \%$ to yield a statistically significant result for the primary outcome. This population was recruited as a convenience sample of children with a similar socio-economic background. Of the 1382 subjects that provided informed consent, 198 of these subjects were not eligible for analysis due to one or more excluded criteria, and another 83 subjects were not eligible for data analysis due to incomplete data collected. The final sample size for analysis was 1101 subjects. Of this sample, $55.8 \%(n=614)$ were boys and $44.2 \%(n=487)$ were girls, and this distribution difference was not statistically significant $(\mathrm{p}=0.415)$. Excluded criteria for subjects were: history of acute or chronic conditions that may affect feeding habits or nutritional status; any chronic medications that may modify appetite or nutritional supplements, including iron, on a daily basis for more than two weeks during the past month prior to screening; or current treatment for a feeding problem, i.e., nutritional intervention or nutritional supplementation.

\section{Measures}

\section{Study Procedures}

This survey study and documents therein were approved by school authorities, and the Fiscalía de Menores de Madrid (Minor's Prosecuting Office in Madrid) was informed of the study. An envelope was sent home from school with the student with forms for the parents to fill out. These initial forms collected the following information: written informed consent, medical history and current medications, dietary history, eating behaviors and demographics (e.g., age, gender, household information). If a parent agreed for their child to participate in the survey via consent form, three more documents were sent home to collect survey data. These were the three-day food record, a family irritability questionnaire, and the Children's Eating Behaviour Questionnaire [11], which were reviewed by study personnel for accuracy, legibility and completeness when they were returned to the school. School personnel were trained by the contract research organization Effice (CRO) on the protocol, proper use of worksheets, informed consent procedures, maintenance of essential study documents and any other study procedures at, or before, study initiation. Height and weight for subjects were measured by the CRO personnel at the schools. The teachers were trained by the CRO on the recording of nutritional data for the lunch meal, and then teachers trained participating parents on the food diaries. The food diaries collected the types of foods, quantities using household measurements, and cooking techniques. To determine the nutrient intake of subjects, three-day food records were filled out for two days during the week plus one weekend day. Teachers and CRO personnel monitored the food intakes in the school for the 2 week-days for those children having lunch in the school. Parents filled out the dietary intake for the weekend along with other meals outside of school. A registered dietitian converted quantities of foods consumed into gram quantities. These quantities of food were then analyzed by food nutrition software DIAL Version 2.12. The average daily quantities of foods in specific food groups were compared with the Spanish recommendations [12]. To measure attention, the Cumanin test for attention was used [13]. This test was administered by the study psychologist and CRO personnel at the school. This is a short test in duration and was administered individually to all subjects in the sample.

\section{Classification of Subjects}

In this survey, the primary criteria for classifying a child as a picky eater was consumption of less than $65 \%$ of the average daily recommended intake for at least four out of six food groups [12]. The six food groups are meat (including fish and eggs); vegetables; fruit; dairy; starch carbohydrates (pasta, bread, rice); and legumes [12]. The gram weights of foods consumed via three-day food records were used to calculate the percentage of recommendations met. Additionally, the Children's Eating Behaviour Questionnaire (CEBQ) was used to classify subjects as 'fussy' eaters, 'emotional undereaters', etc [11].

\section{Statistical Analysis}

Data from a pilot study including data from the first enrolled school was to be analyzed to determine the necessity for adjusting the original sample size to reach study objectives. After consideration of the interim data, the sample size did not need to be recalculated. In the study pilot and total sample analysis, adjustments for type I errors were conducted. Quantitative variables were described using the following statistics: number of cases, mean, standard deviation, minimum, maximum, median and quartiles. Qualitative variables were described using frequency and percentage.

The three-day food records were analyzed to determine consumption of food groups for PE classification and individual nutrient intakes. To analyze differences in ability to concentrate between PE children and HE children, a Chisquare test was used to compare good positive responses between groups. These responses were also assessed by gender, age and schools by means of stratified and multivariate analysis.

To analyze the differences between picky eating children and healthy ones for protein, energy and micronutrient (Mg, 
Ca, Fe, Zn, folic acid, vitamins A, D, E, $\mathrm{B}_{6}$ and $\mathrm{B}_{12}$ ) intakes derived from subject three-day food records, the student's ttest was used for analysis. The association between eating behaviors measured by the CEBQ [11] and the classification of children with picky eating behavior was assessed in exploratory analyses.

\section{RESULTS}

\section{Demographics}

According to the study definition, picky eating behaviors were present in $18.4 \%$ of the total sample. There were no statistically significant differences in anthropometric data (height, weight, BMI z-scores) or age between $\mathrm{HE}$ and PE children (Table 1). Regarding collected socioeconomic data (number of children in the home, number of persons who contribute financially in the home, and the number of members in the family unit), we found no statistically significant differences between the two groups ( $p$ values $0.366,0.462$ and 0.455 , respectively).

\section{Attention}

There were no differences between the two groups for the attention variable $(\mathrm{p}=0.683)$. However, when data was analyzed according to age, there was a trend starting at 49 months of age with the percentage of PE children below the $20^{\text {th }}$ percentile in Cumanin score (the level when intervention is recommended) increasing up to $50 \%$ of PE children in the $67-78$ month age group scoring below the $20^{\text {th }}$ percentile (Fig. 1). Likewise, when we compared the percentage of children with a Cumanin score below the $20^{\text {th }}$ percentile in the two groups ( $\mathrm{HE}$ and $\mathrm{PE}$ ), the PE group was statistically greater in the 49 to 78 month-old subgroup $(p=0.044)$ (Fig. 2), and this group consisted of 788 individuals, or $70.4 \%$ of the total sample. Of interest, when one reviews the graph in Fig. (1), it can be seen that while not statistically significant $(\mathrm{p}=0.067)$, the number of children at the lower Cumanin percentile is greater in the HE group compared with the PE at the younger ages (43-48 months).

Table 1. Baseline Characteristics of Subjects

\begin{tabular}{|c|c|c|c|}
\hline & Healthy Eaters (HE) & Picky Eaters (PE) & P value \\
\hline \hline Age in years & $4.73 \pm 0.9$ & $4.71 \pm 0.9$ & 0.121 \\
\hline Weight in kg z-score & $0.58 \pm 1.07$ & $0.58 \pm 1.15$ & 0.964 \\
\hline Height in cm z-score & $0.45 \pm 1.20$ & $0.40 \pm 1.37$ & 0.615 \\
\hline BMI (body mass index) z-score & $0.31 \pm 1.11$ & $0.36 \pm 114$ & 0.620 \\
\hline
\end{tabular}

All values are mean \pm standard deviation; data was analyzed via t-test for equality of means

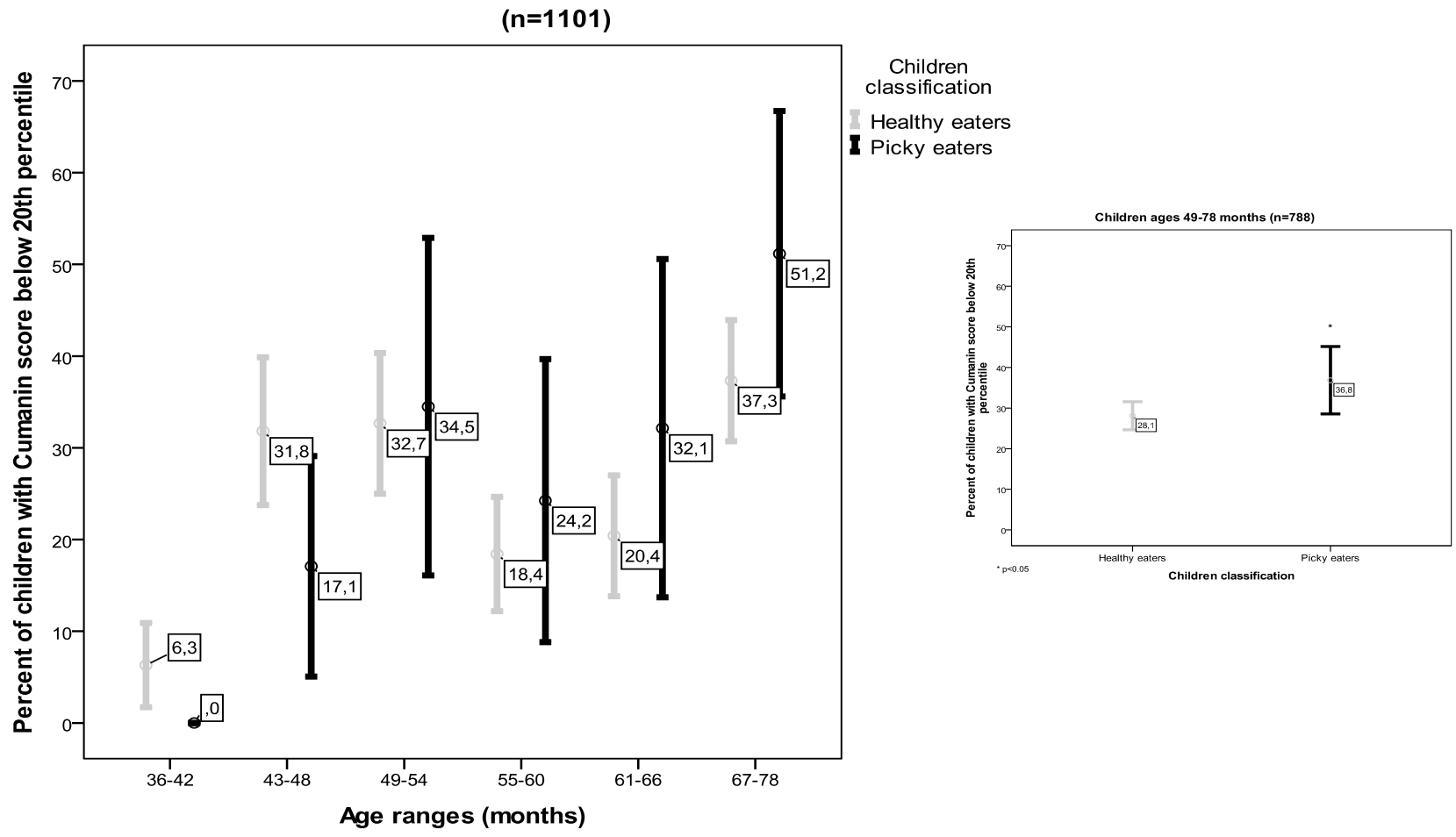

Fig. (1). Attention measures by age ranges. 


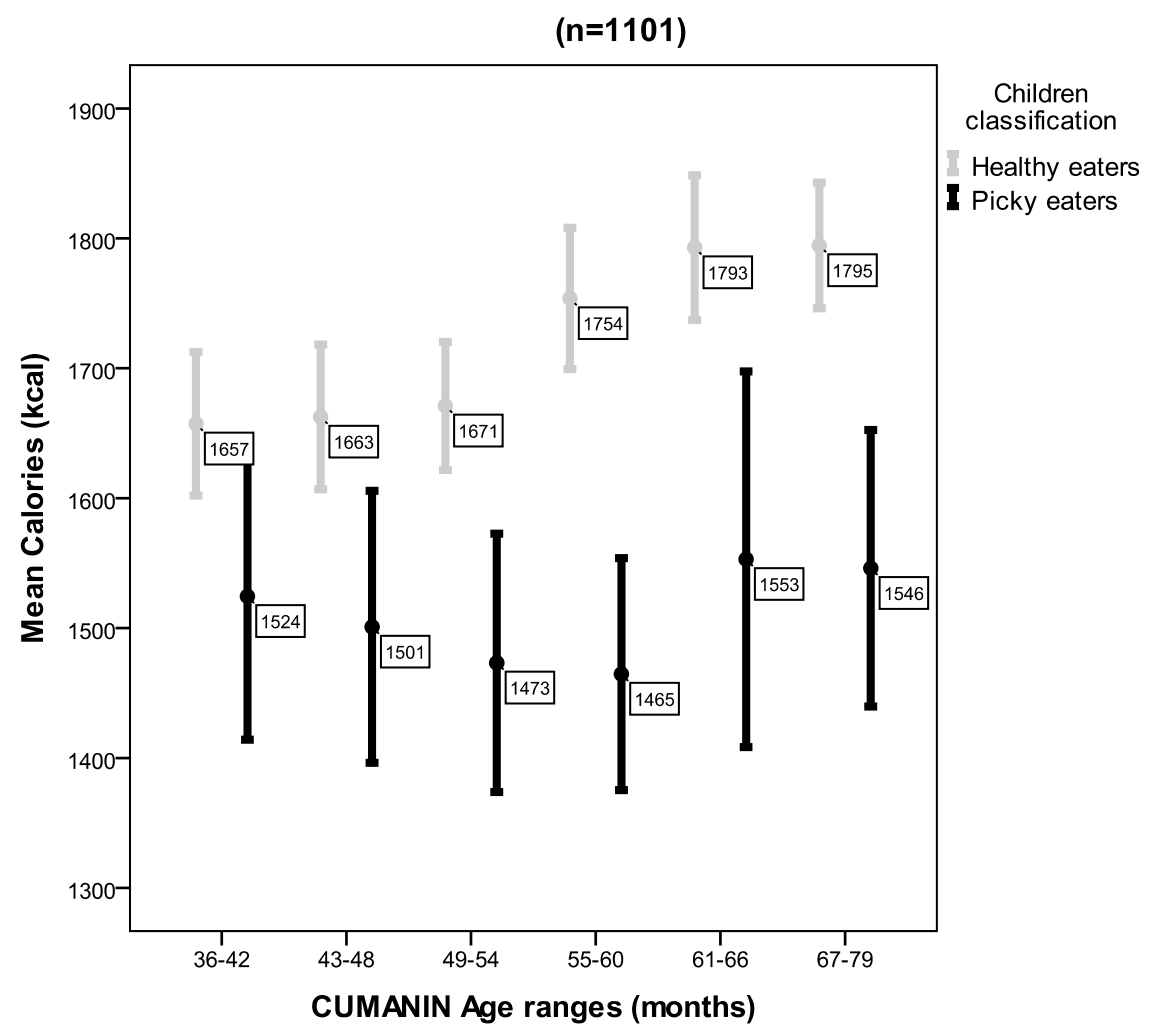

Fig. (2). Caloric intakes by group and age classification.

(Analyzed via t-test for equality of means)

\section{Nutrient Intake}

As expected, the intakes in the PE children group were significantly lower when compared with the HE children group, both for energy as well as for all nutrients analyzed with the exception of vitamins $\mathrm{D}$ and $\mathrm{B}_{12}$, which was also lower but not statistically significant (Table 2 ). If one considers the analysis of energy intake, we can clearly see the intake of PE children is statistically lower for all age ranges analyzed when compared with that in the HE group. One interesting observation is the increase in caloric intake with age in HE children, but a constant intake regardless of age in the PE group (Fig. 2). When we compare the nutrient intake data with the estimated average requirements (EAR) published by the National Academy of Science for children 4-8 years of age, the PE group does not meet the recommended daily intakes for folic acid (94\%), vitamin E (92\%) and vitamin D (21\%) (Table 3). Children in the HE group only met $26 \%$ of the recommended intake for vitamin D. If one would compare the PE data versus the DRI recommendations in Table 3, deficiencies in iron (81\%); calcium (81\%); folic acid (76\%); vitamin E (79\%); and vitamin D (14\%) can be found. The intake for n-3 and n-6 fatty acids was analyzed but not presented here due to low intake numbers for both groups of children, due to a lack of data available for many products marketed in Spain. All children with a Cumanin score $<20^{\text {th }}$ percentile were also observed to have a statistically lower consumption of two distinct food groups: dairy $(p=0.021)$; fish, meat and eggs $(p<0.001)$.

\section{Children's Eating Behaviour Questionnaire}

The data for the Children's Eating Behaviour Questionnaire showed significant differences between the two groups of children for five out of eight eating behavioral categories - enjoyment of food, desire to drink, satiety responsiveness, slowness in eating, and fussiness (Table 4). This data is measured by a validated 35-question tool with a 1-5 point Likert scale measuring from "never" to "always". All questions are related to eating behaviors that are then organized under the eight specific categories. While this data was not a primary or secondary outcome for the study, it does show that picky eating might be defined by these categories and their related questions for eating behaviors.

*Please note that family irritability data will be presented elsewhere.

\section{DISCUSSION}

Our definition for picky eating was established with actual nutrient intakes of children ages 3-6 years via threeday food records, which are often considered a gold standard in terms of average dietary intake for an individual. Our definition of picky eating was average daily intake that did not meet at least $65 \%$ of the published recommendations for at least four out of six defined food groups in Spain [12]. For our sample of 1101 children, $18.4 \%$ met the definition for $\mathrm{PE}$. Because there is currently no validated definition for the behavior of picky eating, and the majority of current research 
Table 2. Energy and Nutrients by Group Classification

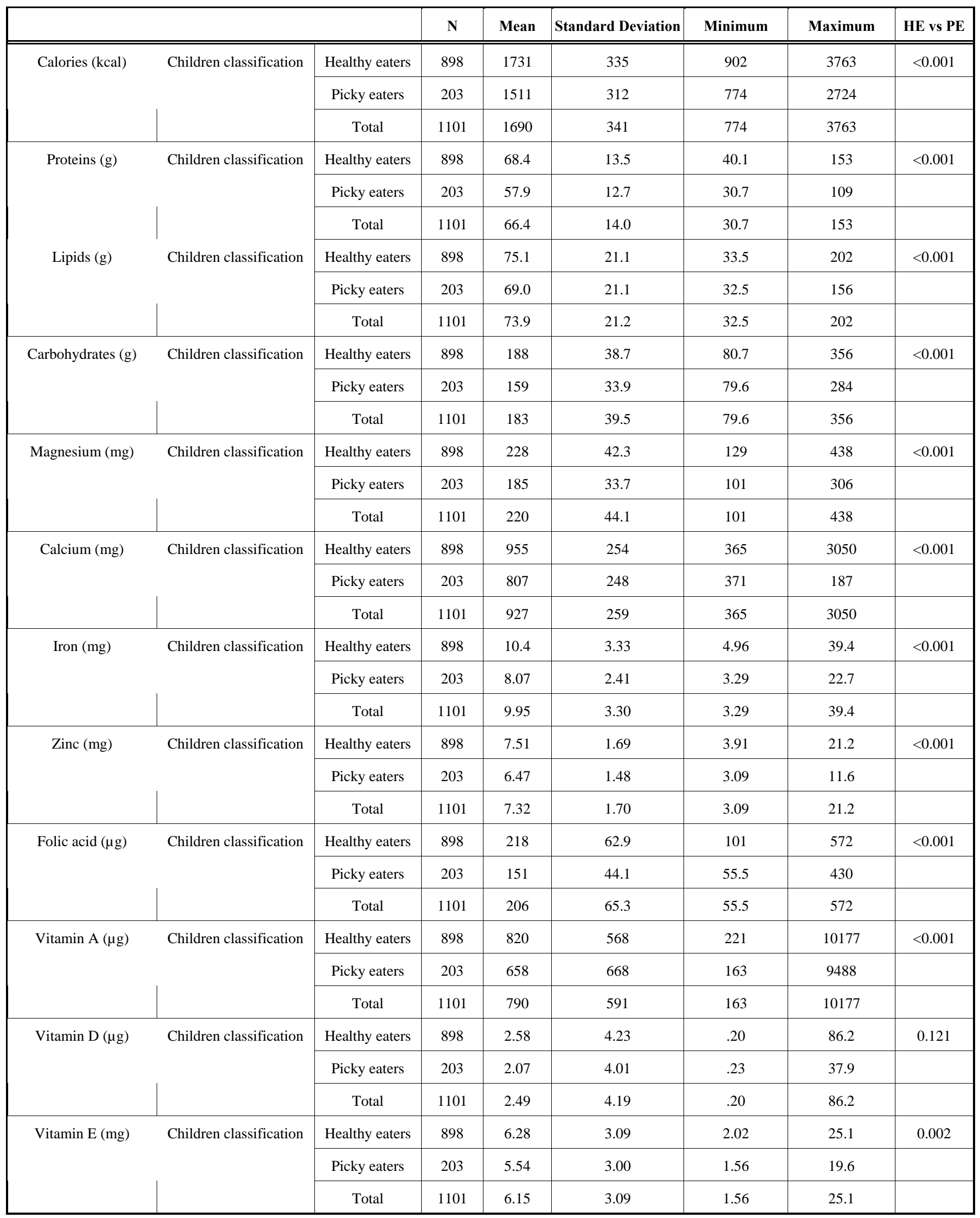


Table 2. Contd.....

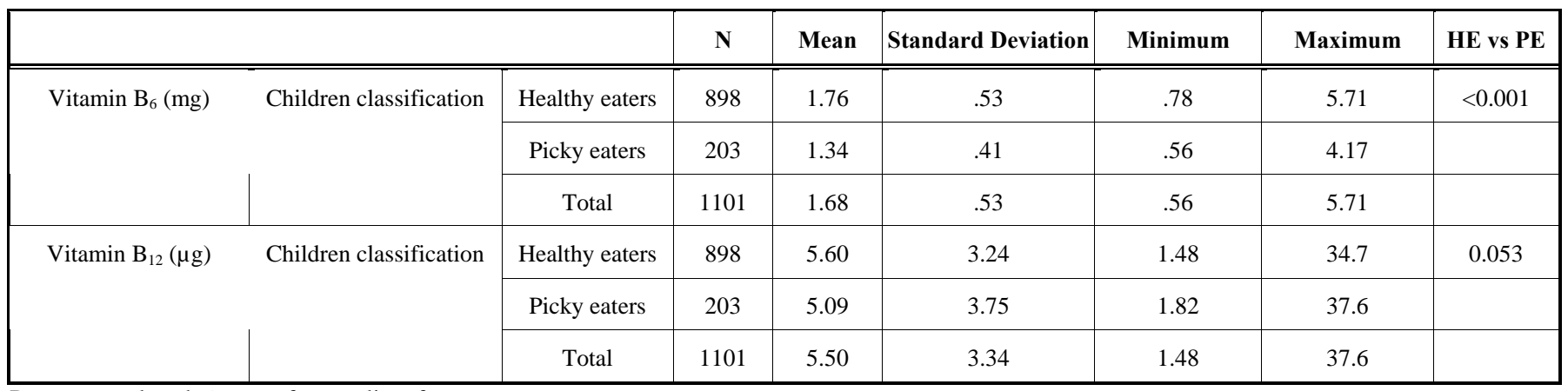

Data was analyzed via t-test for equality of means.

Table 3. Comparison of Study Nutrient Data with Daily Recommendations of Nutrients

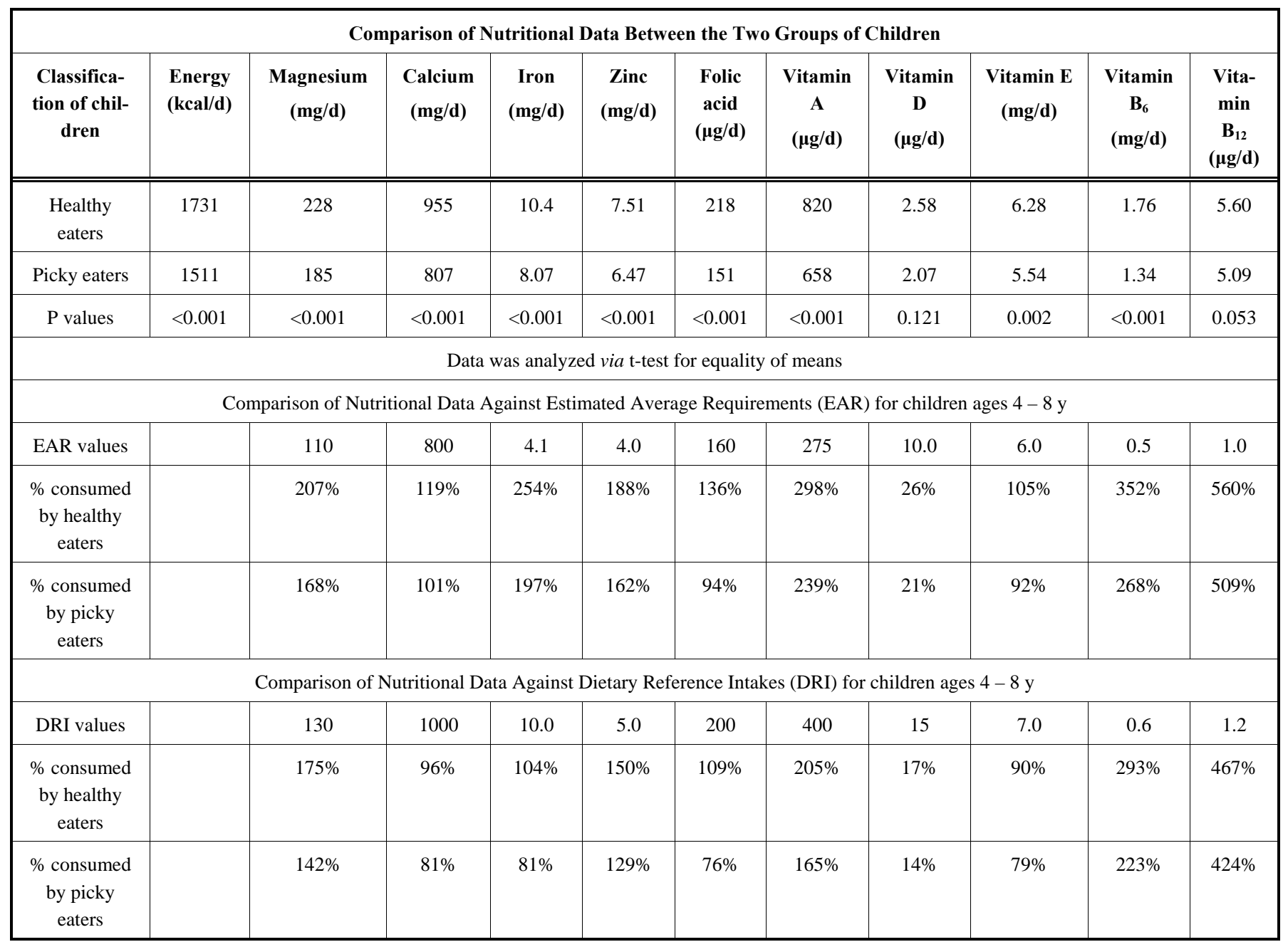

EAR and DRI reference values from Food and Nutrition Board, Institute of Medicine, National Academies (reports with values can be accessed at www.nap.edu)

available on this category of feeding disorders is subject to highly subjective data, i.e. parental opinions about dietary habits, it was necessary for picky eating to be defined by nutritional data. However, nutritional data can sometimes be called in to question with analyses demonstrating the commonality of over-reporting and under-reporting in food records or dietary recall [19-22]. The study authors agree that food records can be faulty and highly subjective, but chose nutritional assessment as a more objective means to define the picky eater child.

In Spain, there are many tools to evaluate attention and memory in children, but the Cumanin battery of tests is the only one that is validated for children ages 36-78 months in age. Cumanin encompasses many areas of child development that coincide with the start of formal schooling such as psychomotricity (motor skills), language, attention, spatial struc- 
Table 4. Children's Behaviour Questionnaire Data

\begin{tabular}{|c|c|c|c|}
\hline \multirow{2}{*}{ Eating Behavior Category } & \multicolumn{2}{|c|}{ Subject Classification } & \multirow{2}{*}{ P-value } \\
\cline { 2 - 4 } & Healthy Eater (HE) & $2.04 \pm 0.67$ & 0.147 \\
\hline \hline Food Responsiveness & $2.12 \pm 0.72$ & $1.74 \pm 0.51$ & 0.821 \\
\hline Emotional overeating & $1.73 \pm 0.51$ & $3.25 \pm 0.70$ & $0.003 *$ \\
\hline Enjoyment of food & $3.42 \pm 0.70$ & $2.45 \pm 0.84$ & $<.001 *$ \\
\hline Desire to drink & $2.25 \pm 0.78$ & $2.93 \pm 0.63$ & $0.001 *$ \\
\hline Satiety responsiveness & $2.72 \pm 0.65$ & $2.00 \pm 0.58$ & $0.012 *$ \\
\hline Slowness in eating & $1.88 \pm 0.60$ & $3.20 \pm 0.88$ & 0.114 \\
\hline Emotional undereating & $3.09 \pm 0.89$ & $3.11 \pm 0.88$ & $<0.001 *$ \\
\hline Fussiness & $2.78 \pm 0.81$ & \\
\hline
\end{tabular}

All values are mean \pm standard deviation; data was analyzed via t-test for equality of means

* Denotes values for $\mathrm{p}<0.05$

ture, visual perception, memory, rhythm-temporal structure and laterality. This tool is widely used in Spain for various assessments in children such as psychological, medical, educational, and neurological. Attention is measured through a subject's recognition of objects, by measuring the ability to focus his or her attention, which is called selective attention or concentration, which is very important in an academic environment. With this sample of 1101 children, we did not see a significant difference between the healthy eaters and picky eaters for the attention measure, except in the older age ranges. This might be explained by a cumulative effect of PE behaviors and their ensuing nutritional deficits, which could also help explain why the healthy eater group had worse attention scores in the younger age group (43-48 mo). The sampling for this study was conducted in the fall season when children began school after summer break, so it is possible that the younger children were still adjusting to the school environment, and a bias was introduced to the attention measure for this group.

Because the PE group was defined by sub-optimal intake of daily recommendations $(<65 \%$ of the average daily recommended intake for at least four out of six food groups), it was expected that differences would be found between the two groups. Although the $\mathrm{PE}$ children as a group met or exceeded the estimated average requirements (EAR) for most of the micronutrients analyzed, as other authors have already published [14], some subjects have a highly reduced intake of some basic food groups such as vegetables and fruits and, also, do not meet caloric intake recommendations. Continued picky eating behavior in the manner of this study could lead to prolonged nutritional deficiencies that may affect attention or school performance after a period of time. It is important to note that nutrient comparisons were made against daily intake recommendations for children ages 4-8 y and this survey study collected data on children ages 3-6 y (Table 3).

Wardle and her colleagues have used the Children's Eating Behaviour Questionnaire extensively, especially in the area of obesity research [15-18]. The data generated in the present study with this specific questionnaire is of inter- est with regards to classifying children with inadequate eating behaviors. Future studies utilizing the tool may increase it's usefulness in the area of picky eating.

\section{CONCLUSION}

While the primary outcome was not achieved in this study, there were lower scores in attention for the picky eater children in the older age groups compared with the healthy eaters. And so, the older ages (4-6 y) should be further investigated, and possibly studied with a more narrow definition for picky eating, including children with more moderate or severe picky eating behaviors. This study is important for defining picky eating by using objective rather than the subjective means used in other pediatric studies, and also for providing valuable information on the current eating behaviors and nutrient intakes of healthy children between ages 3 and 6 years in Madrid, Spain.

\section{CONFLICT OF INTEREST}

This survey study was sponsored by Abbott Nutrition, and authors JAW, BJM and RRD are employees of Abbott Nutrition.

\section{ACKNOWLEDGEMENTS}

We thank the study subjects and their parents for participation in this trial, along with the school teachers and administration for their willingness to allow the research to be conducted within the school systems.

\section{REFERENCES}

[1] Mascola AJ, Bryson SW, Agras WS. Picky eating during childhood: a longitudinal study to age 11 years. Eat Behav 2010; 11: 253-7

[2] Galloway AT, Fiorito L, Lee Y, Birch LL. Parental pressure, dietary patterns and weight status among girls who are picky eaters. J Am Diet Assoc 2005; 105: 541-8.

[3] Dubois L, Famrer A, Girard M, Peterson K, Tatone-Tokuda F. Problem eating behaviors related to social factors and body weight in preschool children: a longitudinal study. Int J Behav Nutr Phys Act 2007; 4: 1-10. 
[4] Lindberg L, Ostberg M, Isacson IM, Dannaeus M. Feeding disorders related to nutrition. Acta Paediatr 2006; 95: 425-9.

[5] Saarilehto S, Lapinleimu H, Keskinen S, Helenius H, Talvia S, Simell O. Growth, energy intake, and meal pattern in five-year-old children considered as poor eaters. J Pediatr 2004; 144: 363-7.

[6] Wright CM, Parkinson KN, Shipton D, Drewett RF. How do toddler eating problems relate to their eating behavior, food preferences, and growth? Pediatrics 2007; 120: 1068-75.

[7] Kar BR, Rao SL, Chandramouli BA. Cognitive development in children with chronic protein energy malnutrition. Behav Brain Funct 2008; 4: 1-12.

[8] Florence MD, Asbridge M, Veugelers PJ. Diet quality and academic performance. J Sch Health 2008; 78: 209-15.

[9] Chatoor I, Surles J, Ganiban J, Beker L, Paez LM, Kerzner B. Failure to thrive and cognitive development in toddlers with infantile anorexia. Pediatrics 2004; 113: 440-7.

[10] Feinstein L, Sabates R, Sorhaindo A, et al. Dietary patterns related to attainment in school: The importance of early eating patterns. $\mathrm{J}$ Epidemiol Community Health 2008; 62: 734-9.

[11] Wardle J, Guthrie CA, Sanderson S, Rapoport L. Development of the children's eating behaviour questionnaire. J Child Psychol Psychiat 2001; 42: 963-70.

[12] Costa CM, Mar LR. Nutrición en el Niño Preescolar y Escolar. En: Tratamiento en Gastroenterología, Hepatología y Nutrición Pediátrica. $2^{\text {nd }}$ ed. 2008: pp. 603-13.

[13] Portellano JA, Mateos R. y Martínez AR, Granados MJ y Tapia A. Cumanin, cuestionario de madurez neuropsicológica infantil. Ed Tea 1999.
[14] Carruth BR, Ziegler PJ, Gordon A, Barr SI. Prevalence of picky eaters among infants and toddlers and their caregivers'decisions about offering a new food. J Am Diet Assoc 2004; 104: S57-S64.

[15] Carnell S, Wardle J. Measuring behavioural susceptibility to obesity: Validation of the child eating behaviour questionnaire. Appetite 2007; 48; 104-13.

[16] Ashcroft J, Semmier C, Carnell S, van Jaarsveld CHM, Wardle J. Continuity and stability of eating behavior traits in children. Eur $\mathrm{J}$ Clin Nutr 2008; 62: 985-90.

[17] Webber L, Hill C, Saxton J, van Jaarsveld CHM, Wardle J. Eating behavior and weight in children. Int J Obes 2009; 33: 21-8.

[18] Webber L, Cooke L, Hill C, Wardle J. Associations between children's appetitive traits and maternal feeding practices. J Am Diet Assoc 2010; 110: 1718-22.

[19] Johansson L, Solvoll K, Aa Bjorneboe GE, Drevon CA. Under- and overreporting of energy intake related to weight status and lifestyle in a nationwide sample. Am J Clin Nutr 1998; 68: 266-74.

[20] Waling MU, Larsson CL. Energy intake of Swedish overweight and obese children is underestimated using a dietary history interview. J Nutr 2009; 139: 522-7.

[21] Black AE, Cole TJ. Biased over- or under-reporting is characteristic of individuals whether over time or by different assessment methods. J Am Diet Assoc 2001; 101: 70-80.

[22] Livingstone MB, Robson PJ, Wallace JM. Issues in dietary intake assessment of children and adolescents. Br J Nutr 2004; 92: S21322. 